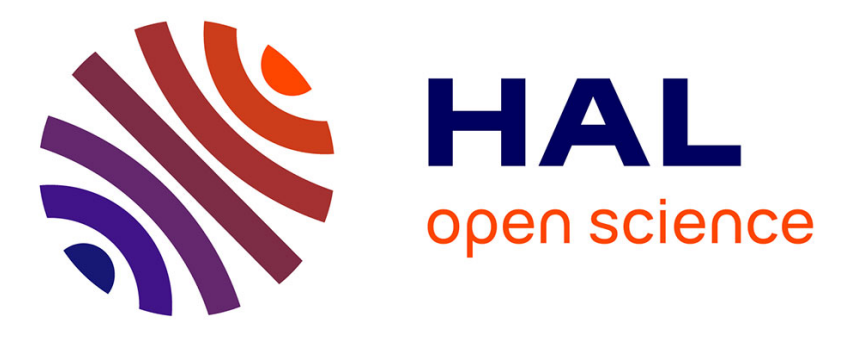

\title{
Development of an immersive simulation platform to study interactions between automated vehicles and pedestrians
}

Lucie Leveque, Thierry Bellet, Jean-Charles Bornard, Jonathan Deniel, Maud Ranchet, Estelle de Baere, Bertrand Richard

\section{To cite this version:}

Lucie Leveque, Thierry Bellet, Jean-Charles Bornard, Jonathan Deniel, Maud Ranchet, et al.. Development of an immersive simulation platform to study interactions between automated vehicles and pedestrians. CHIRA 2020, 4th International Conference on Computer-Human Interaction Research and Applications, Nov 2020, Budapest, Hungary. pp.249-254, 10.5220/0010125002490254. hal-03166267v2

\section{HAL Id: hal-03166267 \\ https://hal.science/hal-03166267v2}

Submitted on 11 Mar 2021

HAL is a multi-disciplinary open access archive for the deposit and dissemination of scientific research documents, whether they are published or not. The documents may come from teaching and research institutions in France or abroad, or from public or private research centers.
L'archive ouverte pluridisciplinaire HAL, est destinée au dépôt et à la diffusion de documents scientifiques de niveau recherche, publiés ou non, émanant des établissements d'enseignement et de recherche français ou étrangers, des laboratoires publics ou privés. 


\title{
Development of an Immersive Simulation Platform to Study Interactions between Automated Vehicles and Pedestrians
}

\author{
Lucie Lévêque ${ }^{1}$, Thierry Bellet ${ }^{1}$, Jean-Charles Bornard ${ }^{2}$, Jonathan Deniel ${ }^{1}$, Maud Ranchet ${ }^{1}$, \\ Estelle De Baere ${ }^{1}$ and Bertrand Richard ${ }^{1}$ \\ ${ }^{1}$ Laboratory of Ergonomics and Cognitive Sciences Applied to Transport, Université Gustave Eiffel, Lyon, France \\ ${ }^{2}$ Engineering Systems International (ESI) Group, CIVITEC, Lyon, France
}

Keywords: Vehicle/Pedestrian Interactions, Automated Vehicle (AV), Simulation, Human-centred Design.

\begin{abstract}
The importance of informal communication between manual vehicles drivers and pedestrians in order to prevent misinterpretation, and thus accidents, in road-crossing situations has been widely shown in the literature. Such crucial communication consequently raises the issue of the introduction of automated vehicles (AVs) on the roads, in which case the status of the driver becomes less obvious. In this paper, we present a novel simulation platform, the V-HCD, allowing the conduct of immersive experimentations, both from the pedestrian's and the driver's point of view. This platform will be used to study the acceptance of the automated vehicle for the European SUaaVE project, and further to support the human-centred design of a future empathic AV.
\end{abstract}

\section{INTRODUCTION}

With the rapid development of new technologies and automation, the introduction of automated vehicles (AVs), or connected automated vehicles (CAVs), i.e., cars without active drivers, offers a strong potential to increase both traffic safety and accessibility. In fact, in the near future, the traffic system will be shared between fully automated vehicles, partially automated vehicles, and manually driven vehicles (Litman, 2020). However, it should be noted that automated vehicles (i.e., levels 3 to 5 of driving automation (SAE, 2018)) will not only coexist with more conventional vehicles (i.e., levels 0 to 2 of driving automation), but also with vulnerable road users, including pedestrians. Generally, pedestrians are considered an important indicator of a society's health and safety. With a view to contribute to a safe traffic system, and consequently to increase the acceptance of $\mathrm{AVs}$, one key challenge is therefore to investigate how the latter interact with pedestrians.

To achieve safe interactions, manual vehicle drivers and pedestrians need to share their understanding and awareness of the traffic situation (Endsley, 1995; Bellet et al., 2009). Otherwise, critical conflicts may occur; $21 \%$ of fatal road traffic accidents happen to involve pedestrians (WHO, 2015). It is crucial to account that misinterpretation of others' intentions is one of the main causes of accidents involving pedestrians (Habibovic et al., 2012). This is particularly true in the case of road crossing decision-making, and especially when priority rules are unclear (e.g., absence of zebra crossing). In such a context, pedestrians and manual drivers frequently interact using non-verbal communication to clarify their intentions. Several studies have shown the importance of this informal communication in the literature.

For instance, Schmidt et al. found that pedestrians who want to cross a street tend to look at the approaching vehicle to get acknowledgement from the driver; if the driver returns their eye contact, pedestrians assume that they have been seen and that they have achieved a mutual understanding (Schmidt et al., 2009). Similarly, Sucha et al. found that pedestrian's decision to cross, as well as their feeling of safety, are directly impacted by various signals provided by the driver, like eye contact, postures, waving hand, or flashing lights (Sucha et al., 2017). Such conclusions were also drawn by Rasouli et al. who showed that the most prominent signal to transmit pedestrians' crossing intention is looking, or at least glancing, towards oncoming traffic (Rasouli et al., 2017). Finally, in their study, Schneemann et al. found that, when pedestrians interact with vehicles at low speed, they tend to rely on eye contact with the driver; whereas, at faster speed, they generally base 
their decisions on the dynamics of the vehicle (Schneemann et al., 2017).

To summarise, all these studies clearly indicate that an active communication between pedestrians and manual vehicle drivers is a crucial element to manage situational risks, to support pedestrians' decisionmaking, and to increase their safety while crossing roads. A key concern regarding the introduction of automated vehicles public roads is therefore due to the changing status of the drivers. Indeed, AVs may negatively impact interactions with pedestrians as they will not be able to rely on cues from drivers' behaviours anymore, potentially leading to uncertainty and mistrust (Vissers et al., 2017). Malmsten Lundgren et al. suggested that the introduction of automated vehicles in the urban context may lead to a notable change in how pedestrians experience AVs compared to conventional vehicles (Malmsten Lundgren et al., 2016). In their study, pedestrians rated eye contact with a driver as promoting safe interaction; whereas apparent driver distraction in an AV (e.g., phoning or reading the newspaper) tended to increase pedestrians' anxiety.

\section{RESEARCH OBJECTIVES}

Regarding automated vehicles, it should be noted that one key challenge is not only to study the acceptability (i.e., before use), but also the acceptance (i.e., after use) (Schade et al., 2003; Distler et al., 2018). However, automated and/or autonomous vehicles are not commonly traveling on European roads yet. Therefore, the only way to be able to study the aforesaid acceptance is by using new generation immersive simulation tools, which allow users to plan for the future thanks to virtual reality (Kyriadikis et al., 2019). By developing such immersive simulation environments, it makes it possible to invite real humans to practically experience future technologies and situations. Investigating the adequacy of future systems to end users' needs, exploring potential risks, and evaluating the acceptance then become conceivable.

The in-depth study of how pedestrians and automated vehicles interact with each other is a crucial issue for the SUaaVE (SUpporting acceptance of automated VEhicle) project. In this context, developing an immersive platform gathering such virtual reality tools would allow the people of today to experience the AVs of tomorrow. In order to reach this goal, Université Gustave Eiffel (ex-IFSTTAR) and ESI/CIVITEC decided to create such an immersive simulation platform for the SUaVVE project, through a pre-existing tool.

\section{DESIGN AND DEVELOPMENT OF A V-HCD PLATFORM}

With a view to better understand and integrate users' needs in the design of innovative advanced driving aid systems (ADAS), IFSTTAR and ESI Group jointly developed a virtual human-centred design platform; the V-HCD (Bellet et al., 2012, 2018). As a virtual simulation toolbox, the V-HCD is able to handle human-based simulation, that is to say based on a virtual driver model, to virtually assess accident risks as well as the potential benefits of future ADAS.

The V-HCD integrative platform is made of two main components: a virtual driver, and a virtual prototyping platform. The virtual driver is based on the cognitive model COSMODRIVE, i.e., COgnitive Simulation Model of the DRIVEr (Bellet et al., 2009). The virtual prototyping platform, named ESI Pro$\mathrm{SiVIC}^{\mathrm{TM}}$, integrates simulated infrastructures, road users, vehicle dynamics, and multi-technology perception sensors (Gruyer et al., 2006).

In a recent study, Bellet et al. (Bellet et al., 2019) used this platform for the human-centred design of a driver monitoring system, with a view to identify risks of collision caused by visual distraction during driving, and in charge to manage human-machine interactions in real time. Figure 1 represents an example of simulation with the COSMODRIVE model implemented in the V-HCD, to simulate the effects of visual distraction on the driver's situation awareness.

In this previous project, the V-HCD was used in the initial stages of the design process, during which there was no prototype of the future system, and therefore no experimentation with real humans nor user testing was possible. In such an early stage, making use of a user model, such as COSMODRIVE, can enable a better comprehension of the risks due to visual distraction depending on the situational context. COSMODRIVE indeed allowed the simulation of various driving scenarios with different levels of driver distraction in order to identify the most critical situations, as illustrated in Figure 2. These critical scenarios were then used as 'reference use cases' (Bellet et al., 2019) to consequently specify functionality to embed in future ADAS with a view to prevent accidents. 

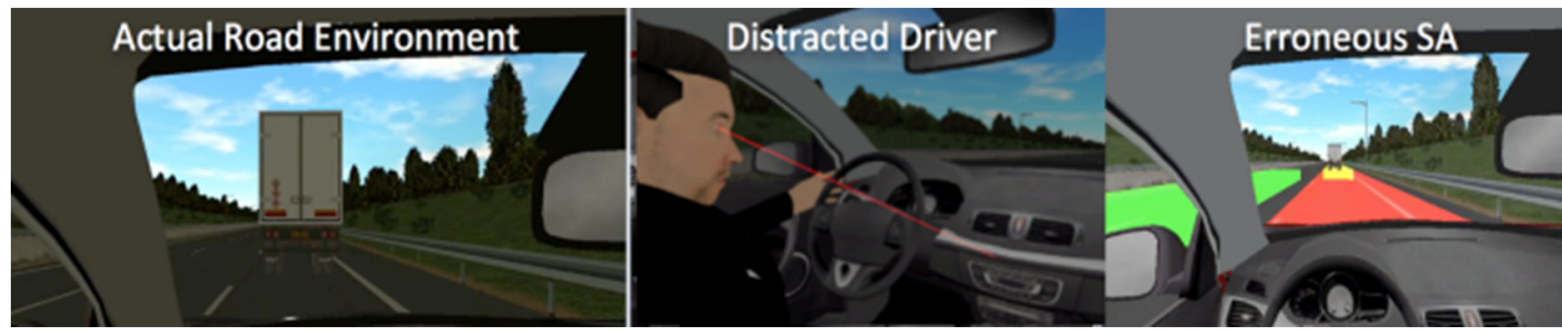

Figure 1: Illustration of the simulation of drivers' visual distraction effects with the V-HCD.

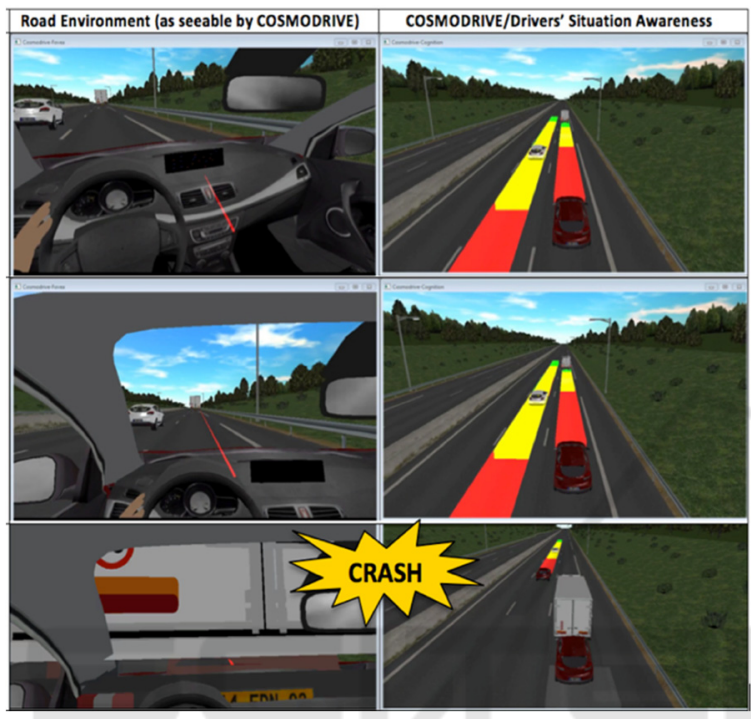

Figure 2: Illustration of an example of simulated accident risks due to visual distraction with the V-HCD platform.

Beyond this early use, the V-HCD can also be used at more advanced stages of the design process. Indeed, as soon as models and/or real or virtual prototypes of the future ADAS are available, the VHCD can be used as an integrated simulator of: (1) the ADAS, (2) the road environment, and (3) the vehicle. Then, real humans can sit in the vehicle to put themselves in the shoes of future users of this ADAS, and consequently have a realistic experience of this future technology before it becomes available on real cars.

In the framework of the SUaaVE project, the objective was to make changes to the original V-HCD platform, in order to focus on the study of AVpedestrians interactions and, more precisely, on the acceptance of such automated vehicles. As introduced in the previous section, the latter are not traveling on the roads yet; therefore, the only way to investigate acceptance is through the use of virtual immersion.

It is with this in mind that the new version of the V-HCD platform was designed and developed for the SUaaVE project, i.e.: to allow users today to plunge into an immersive experience of interaction with an autonomous vehicle, either from the driver/passenger point of view interacting with a virtual pedestrian, or from the pedestrian point of view, willing to cross in front of an AV.

Figure 3 describes this perspective by presenting a short scenario where a pedestrian is crossing the street while an automated vehicle is approaching.

More precisely, Figure 3 (view 1) shows the designer's overview, where the parameters (e.g., cars colours, distances, speeds, accelerations) of each object can be tuned.

Figure 3 (view 2) represents a possible perspective of the aforementioned scenario, where a human subject becomes the pedestrian. Thanks to a virtual reality (VR) headset, they can turn their head to check for oncoming traffic and take the pedestrian crossing. The avatar settled in the oncoming AV can then be positioned at different places (i.e., front or rear seats), and participate in various activities (e.g., driving carefully, sleeping on the steering wheel, talking to a rear passenger), as illustrated in Figure 4. The objective of these diverse situations is to analyse whether, and to what extent, the decision to cross is modified.

Finally, Figure 3 (view 3) corresponds to a more traditional use of simulation, where a participant takes the place of an occupant of the AV. In such a case, they experience the situation either through the use of a traditional driving simulator cabin, or thanks to a VR headset creating a virtual cockpit. The subject can also multi-task while driving (e.g., reading a book, playing a game). The subject can hence experience diverse AV behavirous, and later express their feelings about each of them. Furthermore, different demeanours can be associated with the pedestrian (e.g., crossing quickly, changing their mind and stepping back).

When implemented simultaneously thanks to the $\mathrm{V}-\mathrm{HCD}$, both perspectives (i.e., as a pedestrian and as an AV occupant (driver or passenger), respectively) can enable two participants to interact with each other in a simulated world. In this instance, the first volunteer sits in the driving simulator, while the second one puts themselves in the position of the 


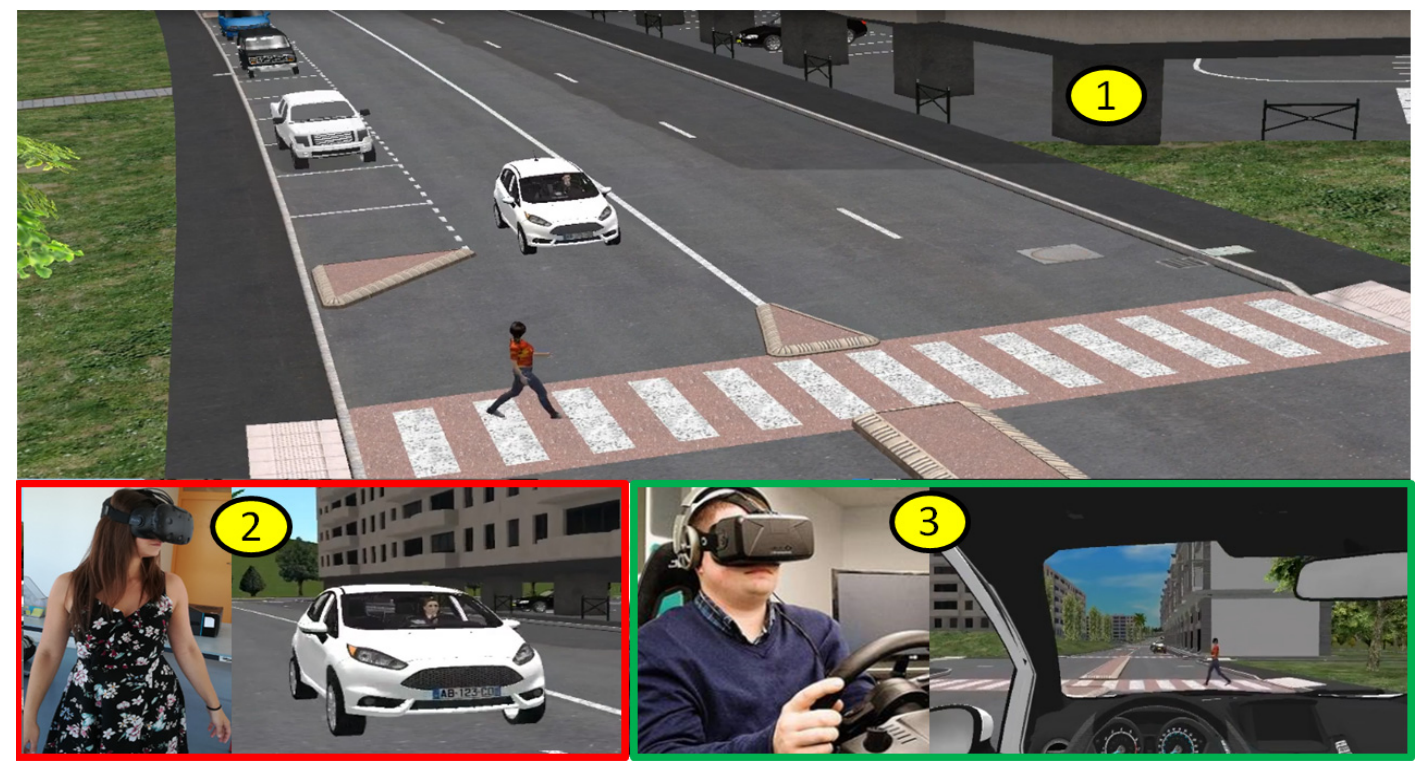

Figure 3: Illustration of an example of scenario implemented on the V-HCD platform to study the interactions between a pedestrian and an AV with a more or less attentive driver.

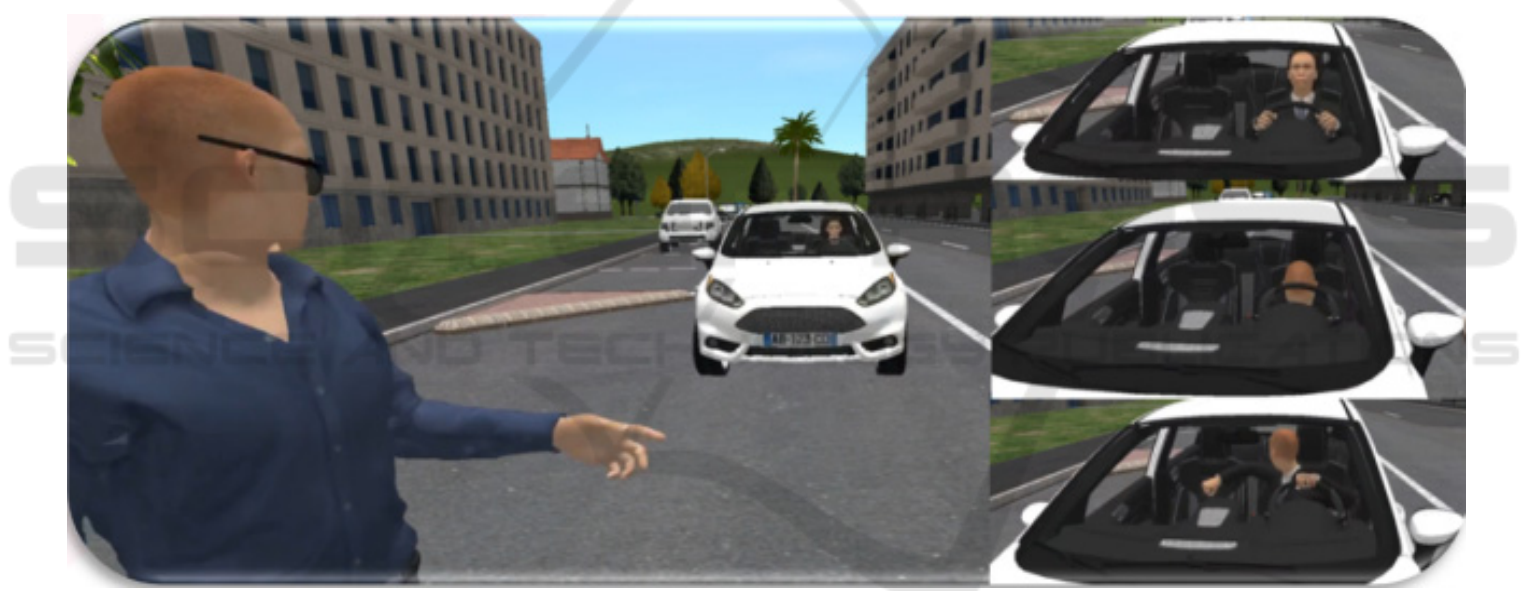

Figure 4: Illustration of examples of driving scenarios implemented on the V-HCD platform to study the interactions between a pedestrian and an AV.

pedestrian by means of the VR headset. Both participants therefore experience the same situation at the same time, but from a different point of view. Each of them can witness the other's behaviour, such as the potential distraction of the AV occupant, or the possible hesitation of the pedestrian before crossing.

The V-HCD platform thereby becomes a set of tools concomitantly integrating automated vehicles (AVs), advanced driving aid systems (ADAS), and different ways to immerse current road users in the future. The AVs are customisable with tailored behaviours originating from cognitive simulation, allowing the fine-tuning of diverse situations while maintaining necessary realism. Simulating ADAS in the immersive experience of road users can further help with the integration of future systems. To summarise, as jointly developed by Université Gustave Eiffel (ex-IFSTTAR) and ESI/CIVITEC, the $\mathrm{V}-\mathrm{HCD}$ allows the testing of AVs and ADAS, as well as of their acceptance by the future end-users.

\section{USE OF THE V-HCD PLATFORM FOR SUaAVE}

In order to study how interactions between pedestrians and automated vehicles may look like in the future, and how these interactions may be affected 
by the AV behaviour, Université Gustave Eiffel will implement a two-phase experiment for the SUaVE project. The first phase will focus on the AV passenger/driver's point of view, whereas the second phase will be dedicated to the pedestrian's point of view.

For the first phase of the experiment, i.e., from the driver/passenger's point of view, participants will experience how the AV reacts when facing diverse pedestrians' road crossing behaviours (i.e., more or less expected and/or critical). For this phase, two different conditions will be considered: with, or without zebra crossing. Several AV behaviours are implemented on the V-HCD to interact with the pedestrian; they will be fully managed and performed by vehicle automation. During each of these alternatives, participants will have the opportunity to use the horn to warn the pedestrian. This way, it will be possible to measure if, and when, they feel that the interaction is becoming too critical, and potentially unacceptable. Participants will afterwards be asked to assess the situational criticality and the reaction of the AV according to the pedestrian's decisions and behaviours. After having experienced all the scenarios, participants will be invited to a semistructured interview to express their suggestions on how an "empathic" automated vehicle should react when interacting with pedestrians, and/or should inform the latter about its decisions and reactions. At this level, the objective of these final questions will be to collect useful information for the future usercentric design of SUaaVE's empathic automated vehicle, namely ALFRED (Automation Level Four and Reliable Empathic Driver).

The second phase of the experiment will be conducted thanks to a simulated environment using virtual reality (VR). Virtual reality indeed allows an immersive, safe, and controlled study of the interactions between a pedestrian and an $\mathrm{AV}$ approaching at different speeds, and with distinct braking behaviours. The second phase of the experiment will focus on the pedestrian willingness or unwillingness to cross the street in front of an automated vehicle, in the absence of zebra crossing, where priority rules are unclear. More precisely, the emphasis will be made on the pedestrian's perceived safety and decision to cross, or not, the road, when interacting with an AV. For this test, participants will be located on a sidewalk, facing a continuous flow of approaching vehicles. First, a randomised number of vehicles, separated with short gaps, will travel without stopping; then, an automated vehicle will appear on the scene. Different behaviours will be implemented in the AV (i.e., in terms of dynamics and ways to stop). The AV occupant, simulated by an avatar, may have different on-board activities (e.g., phoning or discussing with another passenger) or attentive/distracted status, as illustrated in Figure 4. Participants will be asked to use a joystick to assess the safety versus dangerousness of crossing the road from the pedestrian's point of view. Depending on the estimated safety level; the more they will estimate the situation as safe, the further they will have to push the joystick forward. On the contrary, the more the crossing will be assessed as dangerous, the further they will have to pull the joystick backwards. Intending to cross a road being an active decision, keeping the joystick in a neutral position during the whole scenario will correspond to an intention not to cross from the beginning. It will thus be possible to collect the participants' risk assessment in a dynamic way throughout the approaching phase of the $\mathrm{AV}$, and this, whether or not the AV stops.

\section{CONCLUSION AND PERSPECTIVES}

In this paper, we presented a new immersive simulation platform, the V-HCD. This platform was designed to allow users of today to "anticipate the future" by virtually plunging into a simulated, yet realistic, situation of interaction with automated vehicles. This happens to be particularly useful to study the acceptance (after a first use of a technology thanks to virtual reality), and not only the a priori acceptability (i.e., without any practical experience), as well as the relevance of virtual reality to support a cross simulation.

For the SUaaVE (SUpporting acceptance of automated VEhicles) project, this platform will be used in two different, notwithstanding complementary stages. Indeed, a single participant will be able to experience the situation both from the pedestrian's point of view, as well as from the automated vehicle (AV) occupant's point of view. Furthermore, as a result of the efforts performed and using the experience learnt from this project, it is also expected to progress towards an interactive multiusers V-HCD supported by cross-simulation: where several users can experience a given situation and interact in real time, some of them taking the position of pedestrians, and other ones the roles of $\mathrm{AV}$ occupants (i.e., driver or passenger). To conclude, this new approach of virtual cross-simulation opens the gate to different types and multiple synchronised simulations, taking into account humans as end-users and their different mobilities. 


\section{ACKNOWLEDGEMENTS}

This project has received funding from the European Union's Horizon 2020 research and innovation programme under grant agreement No 814999.

\section{REFERENCES}

Bellet, T., Bailly-Asuni, B., Mayenobe, P., Banet, A., 2009. A theoretical and methodological framework for studying and modelling drivers' mental representations. In Safety Science, vol. 47, pp. 1205-1221.

Bellet, T., Mayenobe, P., Bornard, J-C., Gruyer, D., Claverie, B., 2012. A computational model of the car driver interfaced with a simulation platform for future Virtual Human Centred Design applications: COSMOSIVIC. In Engineering Applications of Artificial Intelligence, vol. 25, pp. 1488-1504.

Bellet, T., Richard, B., Laverdure, S., Bornard, J-C., 2018. Use of a cognitive simulation model of the driver to support the Virtual Human Centred Design (V-HCD) of ADAS and automated vehicles. Graz Symposium Virtual Vehicle, Graz, Austria.

Bellet, T., Bornard, J-C., Deniel, J., Richard, B., 2019. Driver modelling and simulation to support the Virtual Human Centred Design of future aids. Human Systems Integration Conference, Biarritz, France.

Distler, V., Lallemand, C., Bellet, T., 2018. Acceptability and acceptance of autonomous mobility on demand: The impact of an immersive experience. Conference on Human Factors in Computing Systems, Montréal, Canada.

Endsley, M., 1995. Toward a theory of situation awareness in dynamic systems. In Human Factors, vol. 37, pp. 3264.

Gruyer, D., Royère, C., Du Lac, N., Michel, G., Blosseville J-M., 2006. SiVIC and RTMaps, interconnected platforms for the conception and the evaluation of driving assistance systems. World Congress and Exhibition on Intelligent Transportation Systems and Services, London, United Kingdom.

Kyriakidis, M., De Winter, J., Stanton, N., Bellet, T., Van Arem, B., Brookhuis, K., Martens, M., Bengler, K., Andersson, J., Merat, N., Reed, N., Flament, M., Hagenzieker, M., Happee, R, 2019. A human factors perspective on automated driving. In Theoretical Issues in Ergonomics Science, pp. 1-27.

Habibovic, A., Davidsson, J., 2012. Causation mechanisms in car-to-vulnerable road users crashes: Implications for active safety systems. In Accident Analysis and Prevention, vol. 49, pp. 493-500.

Litman, T., 2013-2020. Autonomous vehicle implementation predictions. Victoria Transport Policy Institute.

Malmsten Lundgren, V., Habibovic, A., Andersson, J., Lagrström, T., Nilsson, M., Sirrka, A., 2016. Will there be new communication needs when introducing automated vehicles to the urban context? In Advances in Human Aspects of Transportation, pp. 485-497.

Rasouli, A., Kotseruba, I., Tsotsos, J., 2017. Agreeing to cross: How drivers and pedestrians communicate. In IEEE Intelligent Symposium (IV), Washington, USA.

Schade, J., Schlag, B., 2003. Acceptability of urban transport pricing strategies. In Transportation Research Part F: Traffic Psychology and Behaviour, vol. 5, pp. 45-61.

Schmidt, S., Färber, B., 2009. Pedestrians at the kerb: Recognising the action intentions of humans. In Transportation Research Part F: Traffic Psychology and Behaviour, vol. 12, pp. 300-310.

Schneemann, F., Gohl, I., 2017. Analyzing driverpedestrian interaction at crosswalks: A contribution to autonomous driving in urban environments. In IEEE Intelligent Vehicles Symposium (IV), Washington, USA.

Society of Automotive Engineers (SAE), 2018. Taxonomy and definitions for terms related to driving automation systems for on-road motor vehicles. J3016-201806.

Sucha, M., Dostal, D., Risser, R., 2017. Pedestrian-driver communication and decision strategies at marked crossings. In Accident Analysis and Prevention, vol. 102, pp. 41-50.

Vissers, L., Van der Kint, S., Van Schagen, I., Hagenzieker, M., 2017. Safe interaction between cyclists, pedestrians, and automated vehicles: What do we know and what do we need to know? Institute for Road Safety Research, Report R-2016-16.

World Health Organisation (WHO), 2015. Global status report on road safety. 